\title{
AÇÕES DE PROMOÇÃO E PREVENÇÃO À SAÚDE VOCAL DE PROFESSORES: UMA QUESTÃO DE SAÚDE COLETIVA
}

\author{
Promotional and preventive actions for teachers' vocal health: \\ a collective health issue
}

\author{
Karen Fontes Luchesi ${ }^{(1)}$, Lucia Figueiredo Mourão ${ }^{(2)}$, Satoshi Kitamura ${ }^{(3)}$
}

\begin{abstract}
RESUMO
Objetivo: analisar possíveis ações para a prevenção e a promoção da saúde vocal de professores através da análise de interações entre aspectos vocais, ocupacionais e preventivos. Métodos: um estudo qualitativo-quantitativo foi realizado numa escola pública do estado de São Paulo com 26 professores. Desenvolveu-se um programa de aprimoramento vocal que contou com avaliação laringológica, aplicação de questionário e intervenção preventivo-terapêutica em grupo. Para quantificar o grau de associação entre os aspectos vocais, ocupacionais e preventivos, foi utilizado o Teste Qui-quadrado. Resultados: observou-se que a maioria dos professores (exceto dois) referiram algum tipo de queixa relacionada ao uso da voz na profissão. Foram observadas alterações laríngeas em três dos dez professores que se submeteram à avaliação laringológica. Quanto ao estresse, 20 referiram estresse de alguma natureza, e destes, 10 referiram estresse vocal. Treze professores participaram da intervenção. Entretanto, apenas cinco concluíram-na. Houve significância estatística na associação entre a quantidade de sintomas vocais e a participação na intervenção preventivoterapêutica, mostrando que participaram da intervenção, os professores com mais de três sintomas vocais. Também foi observada tendência à associação entre indisponibilidade de tempo e referência a estresse vocal. Conclusão: acredita-se que a saúde vocal do professor esteja amplamente relacionada a aspectos ocupacionais, mesmo não tendo sido encontrado significância estatística em todas as associações investigadas. As ações preventivas devem valorizar as atuações multi ou interdisciplinares, os trabalhos de sensibilização para a percepção da importância da voz no ensino, a identificação e atenuação de fatores de risco, os programas de aprimoramento vocal e a integração entre todos os atores envolvidos com a escola.
\end{abstract}

DESCRITORES: Saúde do Trabalhador; Prevenção de Doenças; Promoção da Saúde; Condições de Trabalho; Voz; Saúde Pública.

(1) Fonoaudióloga; Universidade Estadual de Campinas, UNICAMP, Campinas, SP; Mestre em Saúde Coletiva pela Faculdade de Ciências Médicas da Universidade Estadual de Campinas; Doutoranda em Saúde Coletiva pela Faculdade de Ciências Médicas da Universidade Estadual de Campinas.

(2) Fonoaudióloga; Docente do Curso de Fonoaudiologia da Faculdade de Ciências Médicas da Universidade Estadual de Campinas, UNICAMP, Campinas, SP, Brasil; Doutora em Ciências pela Universidade Federal de São Paulo.

(3) Médico do Trabalho; Docente do Departamento de Medicina Preventiva e Social (Área de Saúde do Trabalhador) da Faculdade de Ciências Médicas da Universidade Estadual de Campinas, UNICAMP, Campinas, SP, Brasil; Doutor em Ciências Médicas pela Faculdade de Ciências Médicas da Universidade Estadual de Campinas.

Conflito de interesses: inexistente

\section{INTRODUÇÃO}

Dados do censo escolar divulgados pelo Instituto Nacional de Estudos e Pesquisas Educacionais Anísio Teixeira (INEP) em 2003 ${ }^{1}$ indicaram que no Brasil há aproximadamente 2,6 milhões de professores, desde a educação básica até o ensino superior.

Tendo em vista que o principal instrumento de trabalho desses profissionais é a voz, a saúde vocal do professor tem sido objeto de estudo de muitos pesquisadores. Todos têm chegado à conclusão de que os riscos para tais trabalhadores desenvolverem distúrbios vocais de ordem ocupacional 
é significativamente alto ${ }^{2,3}$. Pesquisas realizadas nos Estados Unidos encontraram prevalência de problemas vocais, significantemente, maior em professores do que em não professores $(11 \%$ versus $6,2 \%, 15 \%$ versus $6 \%)^{4,5}$. O afastamento de professores, devido a alterações vocais, tem aumentado nos últimos anos ${ }^{7}$.

Ações preventivas vêm sendo estudadas em diversos países ${ }^{6-10}$. Contudo, os dados empíricos a respeito da alteração vocal em professores são limitados, as estratégias de orientação, prevenção e terapia contam com poucas evidências e ainda não têm eficácia cientificamente comprovada ${ }^{11}$.

Alguns estudos nacionais e internacionais investigaram a eficácia de intervenções que visavam tratar e/ou aprimorar o uso da voz de professores, tendo como resultado a melhora significativa na qualidade vocal e na diminuição dos sintomas vocais ${ }^{7,8,10}$. Os mesmos estudos observaram adesão relativamente baixa às intervenções e não houve comprovação da eficácia preventiva das ações realizadas.

A importância da preservação da voz não é reconhecida pela maioria dos professores, que demonstram dificuldades em perceber como problemas, os sinais e os sintomas vocais que apresentam. Na literatura, a falta de percepção dos problemas vocais entre professores, é bastante mencionada ${ }^{9,12-14}$. Há ainda, a aceitação passiva da alteração vocal, pois muitos acreditam ser esta uma consequência natural de sua profissão, além disso, o tempo e o ônus financeiro dispensado ao tratamento da disfonia os tornam relutantes a buscar um acompanhamento apropriado ${ }^{13}$.

Não há dúvidas de que, devido à alta prevalência de problemas vocais e ao grande número de professores no país, são de grande relevância, o estudo e o desenvolvimento de ações preventivas no campo da saúde coletiva, pesquisando não apenas o sujeito, mas também os aspectos envolvidos no desenvolvimento de seu trabalho.

O professor está exposto a fatores de risco ocupacionais, da competência de todos profissionais que se dedicam a zelar pela saúde dos trabalhadores. Acredita-se que a saúde vocal dos professores não consiste na ausência de alterações vocais, mas sim e principalmente, no bem-estar geral destes indivíduos. De acordo com a Carta de Ottawa, a saúde é resultante da ação de fatores políticos, econômicos, ambientais, comportamentais e biológicos ${ }^{15}$.

Com base na aplicação de um programa de aprimoramento vocal numa escola pública, o objetivo do presente artigo foi analisar possíveis ações para a prevenção e a promoção da saúde vocal de professores através da análise de interações entre aspectos vocais, ocupacionais e preventivos.

\section{MÉTODOS}

Com o objetivo de conhecer e compreender melhor o uso da voz e suas implicações desenvolveu-se um estudo quantitativo-qualitativo. O desenho do estudo visou explorar e focalizar a realidade dos professores de forma contextualizada.

O estudo foi desenvolvido numa escola estadual de ensino fundamental, situada na zona de abrangência do Distrito de Saúde Leste do município de Campinas, estado de São Paulo, Brasil. Em 2007, ano do estudo, havia 26 professores presentes na escola (24 do gênero feminino e dois do gênero masculino). O programa de aprimoramento vocal desenvolvido foi oferecido a todos os professores. Utilizou-se como critério de inclusão, ser professor da escola e participar de pelo menos uma das etapas do programa, à escolha individual dos mesmos.

Com o intuito de estudar a prevenção na temática "voz do professor", um programa de aprimoramento vocal foi aplicado na referida escola. O programa era composto por: avaliação laringológica, aplicação de questionário e intervenção preventivo-terapêutica em grupo.

Foi oferecido aos professores, um exame laringológico no Hospital das Clínicas da Universidade Estadual de Campinas (instituição próxima ao local de trabalho dos sujeitos), no Ambulatório de Otorrinolaringologia, para conhecimento de suas condições laríngeas pré-intervenção.

A fim de compreender a demanda vocal dos professores da escola, adaptou-se um questionário anteriormente publicado ${ }^{16}$, sobre aspectos vocais e ocupacionais. Ao questionário foram acrescentadas perguntas abertas sobre a concepção de voz ideal para a profissão de professor, as queixas relacionadas à voz na profissão e sugestões de ações para a saúde vocal. Para o levantamento das sugestões, solicitou-se aos sujeitos que indicassem algum tipo de trabalho interessante para auxiliá-los em relação ao uso da voz no exercício de seu trabalho.

Ao responderem ao questionário os professores referiram se haviam, ou não, notado algum tipo de problema vocal durante os anos de profissão. Quanto aos sintomas vocais, foram orientados a assinalar os que haviam vivenciado ao falar de forma prolongada: fadiga vocal, sensação de coceira na garganta, dor ao falar (na garganta ou região da laringe), garganta raspando, dor no pescoço e/ou nos ombros, engasgo ao falar, falta de ar ou respiração ofegante ao falar, pescoço tenso ao falar, pigarro constante, aumento de volume do pescoço ao falar, garganta seca, voz fraca, falha na voz, dor ao engolir ou outro(s). Também responderam se antes, durante ou depois do período de trabalho 
sentiam estresse de alguma natureza (físico, mental, emocional, vocal).

Para o desenvolvimento da intervenção, foram organizados dois grupos, de 6 e 7 sujeitos. Foram realizados 12 encontros semanais de 1 hora e 30 minutos, na biblioteca e na sala de vídeo da escola, todos coordenados pela mesma fonoaudióloga. Os encontros abordaram itens reconhecidos como fundamentais para uma fonoarticulação adequada e saudável ${ }^{(9,17,22)}$. A saber: noções de anatomia e fisiologia fonatória, saúde vocal (hábitos e cuidados), respiração, coordenação pneumofonoarticulatória, tensão fonatória, articulação, velocidade e modulação da fala, ressonância, projeção vocal, expressividade verbal e não-verbal, aquecimento e desaquecimento vocal. Cada encontro abordou de um a dois itens. Os itens foram desenvolvidos em aulas expositivas, com utilização de recursos áudiovisuais, apresentação oral e diálogo entre os participantes, dinâmicas de grupo e aplicação de técnicas vocais A intervenção foi considerada preventivoterapêutica porque acolheu professores com e sem alterações laríngeas.

A distribuição dos dados foi caracterizada concomitantemente à descrição da amostra, na Tabela 1. Variáveis categóricas foram estabelecidas para análise estatística. Foram analisadas as frequências e porcentagens das variáveis: referência a problema vocal, concepção de voz adequada à profissão, referência a enfermidade, referência a estresse vocal, queixas relacionadas à voz na profissão, indisponibilidade de tempo, quantidade de sintomas vocais, avaliação laringológica, participação na intervenção, conclusão da intervenção. A única variável contínua (quantidade de sintomas vocais) foi categorizada para possibilitar a verificação de seu grau de associação com as demais variáveis categóricas. Para a quantidade de sintomas foram estabelecidas as categorias: 0 a 3 sintomas e 4 ou mais sintomas com base em estudo anteriormente realizado. ${ }^{13}$

O estudo foi conduzido dentro dos padrões exigidos pela declaração de Helsinque e aprovado pelo Comitê de Ética da Faculdade de Ciências Médicas da Universidade Estadual de Campinas em julho de 2007 (CEP №484/2007).

A fim de quantificar o grau de associação entre as variáveis anteriormente citadas, foi utilizado o Teste Qui-quadrado, por meio do software de análise estatística SPSS versão 13.0. A probabilidade $(p)$ menor que 0,05 foi considerada para indicar a significância estatística.

\section{RESULTADOS}

O programa de saúde vocal foi oferecido a toda a população de professores da escola, que era de 26. No entanto por se tratar de programa de adesão voluntária, nem todos escolheram participar de todas as etapas do programa. Os 26 professores responderam aos questionários. Dez professores optaram por submeter-se à avaliação laríngea, 13 participaram da intervenção, mas apenas cinco concluíram-na.

$\mathrm{Na}$ Tabela 1 encontram-se os dados do questionário e da avaliação laringológica. Nos quadros 2 e 3 estão, respectivamente, a frequência e porcentagem dos aspectos investigados e seus graus de associação.

Além dos dados expostos nos quadros é importante relatar que os professores apresentaram em média, 37,5 anos de idade (desvio-padrão $=8,7$ ), 11,9 anos de profissão (desvio-padrão $=6,9$ ) e trabalhavam com carga horária semanal média de 28,6 horas-aula (desvio-padrão $=8,4$ ).

Treze professores $(50,0 \%)$ participaram do grupo de intervenção. Entretanto, apenas cinco $(38,5 \%)$ concluíram-na (Tabela 2). Os profissionais que não participaram ou não concluíram, justificaram o não envolvimento ou a não finalização: $71,4 \%$ (15) por indisponibilidade de tempo, 19,1\% (4) enfermidade e $9,5 \%$ (2) por desinteresse ou por não terem sentido necessidade em participar (Tabela 2).

Foi encontrada significância estatística apenas na associação entre as variáveis: quantidade de sintoma vocal e participação na intervenção. No entanto, observa-se tendência à associação entre as variáveis: indisponibilidade de tempo e referência a estresse vocal (Tabela 3).

Os sintomas vocais mais referidos foram respectivamente: garganta raspando, garganta seca, coceira na garganta e fadiga vocal.

Em relação às ações de prevenção e promoção à saúde, $56 \%$ (14) dos professores sugeriram programas de aprimoramento vocal, $24 \%$ (6) gostaria que houvesse algum tipo de intervenção com os alunos, principalmente relacionado à indisciplina em sala de aula. Dezesseis porcento (4) priorizam a identificação e atenuação dos fatores de risco para doenças relacionadas ao trabalho, $16 \%$ (4) citaram a importância da assessoria de profissionais da equipe de saúde do trabalhador na escola e $8 \%$ (2) referiram que seriam ideais as atuações interdisciplinares (Figura 1). 
Tabela 1 - Descrição da amostra quanto ao gênero, aos sintomas e queixas relacionadas à voz na profissão, à concepção de voz adequada, aos problemas vocais, à avaliação laringológica, à participação na intervenção e à menção a estresse

\begin{tabular}{|c|c|c|c|c|c|c|c|c|c|c|}
\hline \multirow{2}{*}{ Sujeito } & \multirow{2}{*}{ Gênero } & \multirow{2}{*}{$\begin{array}{l}\text { Quantidade } \\
\text { de } \\
\text { sintomas } \\
\text { referidos } \\
\end{array}$} & \multirow{2}{*}{$\begin{array}{c}\text { Queixas } \\
\text { relacionadas } \\
\text { à voz } \\
\text { na profissão }\end{array}$} & \multirow{2}{*}{$\begin{array}{c}\text { Concepção } \\
\text { de } \\
\text { voz } \\
\text { adequada }\end{array}$} & \multirow{2}{*}{$\begin{array}{c}\text { Referência } \\
\text { a } \\
\text { problema } \\
\text { vocal }\end{array}$} & \multirow{2}{*}{$\begin{array}{c}\text { Hipótese } \\
\text { diagnóstica } \\
\text { laringológica }\end{array}$} & \multirow{2}{*}{$\begin{array}{c}\text { Tipo } \\
\text { de } \\
\text { estresse } \\
\text { referido }\end{array}$} & \multicolumn{3}{|c|}{ Intervenção } \\
\hline & & & & & & & & Participação & Justificativa & Conclusão \\
\hline 1 & $\mathrm{~F}$ & 7 & 1 & $\begin{array}{c}\text { Boa } \\
\text { qualidade }\end{array}$ & Sim & Nódulos & $\mathrm{Fi}, \mathrm{Me}$ & Sim & - & Sim \\
\hline 2 & $\mathrm{~F}$ & 14 & 1 & Forte & Sim & Monocordite & $\mathrm{Fi}, \mathrm{Me}, \mathrm{E}, \mathrm{V}$ & Sim & - & Sim \\
\hline 3 & $\mathrm{~F}$ & 5 & 1 & Nítida & Não & $\begin{array}{l}\text { Edema de } \\
\text { Reinke }\end{array}$ & $\mathrm{Fi}, \mathrm{Me}, \mathrm{E}, \mathrm{V}$ & Sim & - & Sim \\
\hline 4 & $\mathrm{~F}$ & 4 & 1 & Firme & Não & $\begin{array}{c}\text { Sem } \\
\text { alteração }\end{array}$ & $\mathrm{Me}$ & Sim & - & Sim \\
\hline 5 & $\mathrm{~F}$ & 5 & 1 & - & Não & $\begin{array}{c}\text { Sem } \\
\text { alteração }\end{array}$ & $\mathrm{Fi}, \mathrm{E}, \mathrm{V}$ & Sim & - & Sim \\
\hline 6 & $\mathrm{~F}$ & 6 & 1 & $\begin{array}{c}\text { Grossa e } \\
\text { forte }\end{array}$ & Sim & $\begin{array}{c}\text { Sem } \\
\text { alteração }\end{array}$ & $\mathrm{Fi}, \mathrm{Me}, \mathrm{E}, \mathrm{V}$ & Sim & IT & Não \\
\hline 7 & M & 0 & 0 & Grave & Sim & $\begin{array}{c}\text { Sem } \\
\text { alteração }\end{array}$ & $\mathrm{Fi}, \mathrm{Me}, \mathrm{E}$ & Sim & ENF & Não \\
\hline 8 & $\mathrm{~F}$ & 8 & 2 & Forte & Sim & $\begin{array}{c}\text { Sem } \\
\text { alteração }\end{array}$ & $\mathrm{Fi}, \mathrm{Me}$ & Sim & $\begin{array}{l}\text { ENF } \\
\text { ENF }\end{array}$ & Não \\
\hline 9 & $\mathrm{~F}$ & 4 & - & Normal & Não & $\begin{array}{c}\text { Sem } \\
\text { alteração }\end{array}$ & Não sente & Sim & ENF & Não \\
\hline 10 & $\mathrm{~F}$ & 3 & 1 & Forte & Não & $\begin{array}{c}\text { Sem } \\
\text { alteração }\end{array}$ & $\mathrm{Fi}, \mathrm{Me}, \mathrm{E}$ & Sim & IT & Não \\
\hline 11 & $\mathrm{~F}$ & 4 & 1 & Forte & Não & - & Me, E & Sim & ENF & Não \\
\hline 12 & $\mathrm{~F}$ & 7 & 2 & Forte & Sim & - & $\mathrm{Fi}, \mathrm{Me}, \mathrm{E}, \mathrm{V}$ & Sim & $\mathrm{IT}$ & Não \\
\hline 13 & $\mathrm{~F}$ & 2 & 1 & Clara & Não & - & Não sente & Sim & $\mathrm{IT}$ & Não \\
\hline 14 & $\mathrm{~F}$ & 0 & 1 & Forte & Não & - & $\mathrm{Fi}$ & Não & INT & - \\
\hline 15 & $\mathrm{~F}$ & 1 & 1 & Forte & Não & - & $\mathrm{Fi}, \mathrm{Me}, \mathrm{E}, \mathrm{V}$ & Não & $\mathrm{IT}$ & - \\
\hline 16 & $\mathrm{~F}$ & 4 & 1 & Forte & Sim & - & $\mathrm{Fi}, \mathrm{Me}, \mathrm{E}, \mathrm{V}$ & Não & IT & - \\
\hline 17 & $\mathrm{~F}$ & 3 & 1 & Forte & Sim & - & Não sente & Não & IT & - \\
\hline 18 & $\mathrm{~F}$ & 0 & 1 & Forte & Não & - & Não sente & Não & IT & - \\
\hline 19 & $\mathrm{~F}$ & 3 & 1 & Agradável & Sim & - & $\mathrm{Fi}$ & Não & IT & - \\
\hline 20 & $\mathrm{~F}$ & 2 & 1 & Forte & Sim & - & $\mathrm{Fi}, \mathrm{Me}, \mathrm{E}, \mathrm{V}$ & Não & $\mathrm{IT}$ & - \\
\hline 21 & $\mathrm{~F}$ & 0 & 0 & Normal & Não & - & Não sente & Não & INT & - \\
\hline 22 & $\mathrm{~F}$ & 2 & 1 & Forte & Sim & - & Não sente & Não & $\mathrm{IT}$ & - \\
\hline 23 & M & 6 & 0 & Grave & Sim & - & $\mathrm{Fi}, \mathrm{Me}, \mathrm{E}, \mathrm{V}$ & Não & IT & - \\
\hline 24 & $\mathrm{~F}$ & 2 & 1 & Nítida & Não & - & $\mathrm{Me}$ & Não & IT & - \\
\hline 25 & $\mathrm{~F}$ & 5 & 1 & Normal & Sim & - & $\mathrm{Me}, \mathrm{E}$ & Não & IT & - \\
\hline 26 & $\mathrm{~F}$ & 8 & 1 & Forte & Sim & - & $\mathrm{Fi}, \mathrm{Me}, \mathrm{E}, \mathrm{V}$ & Não & IT & - \\
\hline
\end{tabular}

Legenda: F= Feminino; $M$ = Masculino. Na coluna "Queixas relacionadas à voz na profissão", 0 = nenhuma dificuldade; $1=$ desconforto ou alteração de qualidade vocal devido à conversa ou indisciplina dos alunos e/ou competição com ruídos internos e externos; $2=$ voz não audível aos alunos. Fi = Físico; $\mathrm{Me}=$ Mental; $\mathrm{E}=$ Emocional; $\mathrm{V}=\mathrm{Vocal} ; \mathrm{IT}=$ indisponibilidade de tempo, ENF= enfermidade, INT = falta de interesse.

\section{DISCUSSÃO}

$\mathrm{Na}$ literatura, observa-se que os professores usualmente referem múltiplos sintomas vocais e os que relatam mais de três sintomas apresentam mais episódios de disfonia, mais dificuldades relacionadas a seu desempenho em sala de aula e maior referência a estresse e frustração ${ }^{13}$.

Neste estudo, nota-se que apesar de referirem múltiplos sintomas vocais e quase todos os professores vivenciarem ou terem vivenciado algum tipo de queixa relacionada à voz na profissão, apenas cinco concluíram a intervenção, e destes, três eram professores com alteração laríngea instalada.
Houve significância estatística na associação entre quantidade de sintomas vocais e participação na intervenção: participaram da intervenção os professores com mais de quatro sintomas vocais. Um estudo com grupos de aprimoramento vocal para professores, realizado na Finlândia, teve como principal motivo para a não adesão à intervenção, a falta de interesse dos professores por não apresentarem alteração vocal aparente ${ }^{6}$. Autores acreditam que a maioria dos professores procura por ajuda, quando a alteração vocal está impactando negativamente na sua atuação ${ }^{8}$. Pesquisadores vêem na implantação de cursos de aprimoramento vocal durante a formação profissional, uma das soluções para maior participação6,7,10. Contudo, percebe-se 
Tabela 2 - Frequência e porcentagem dos aspectos vocais, ocupacionais e preventivos investigados

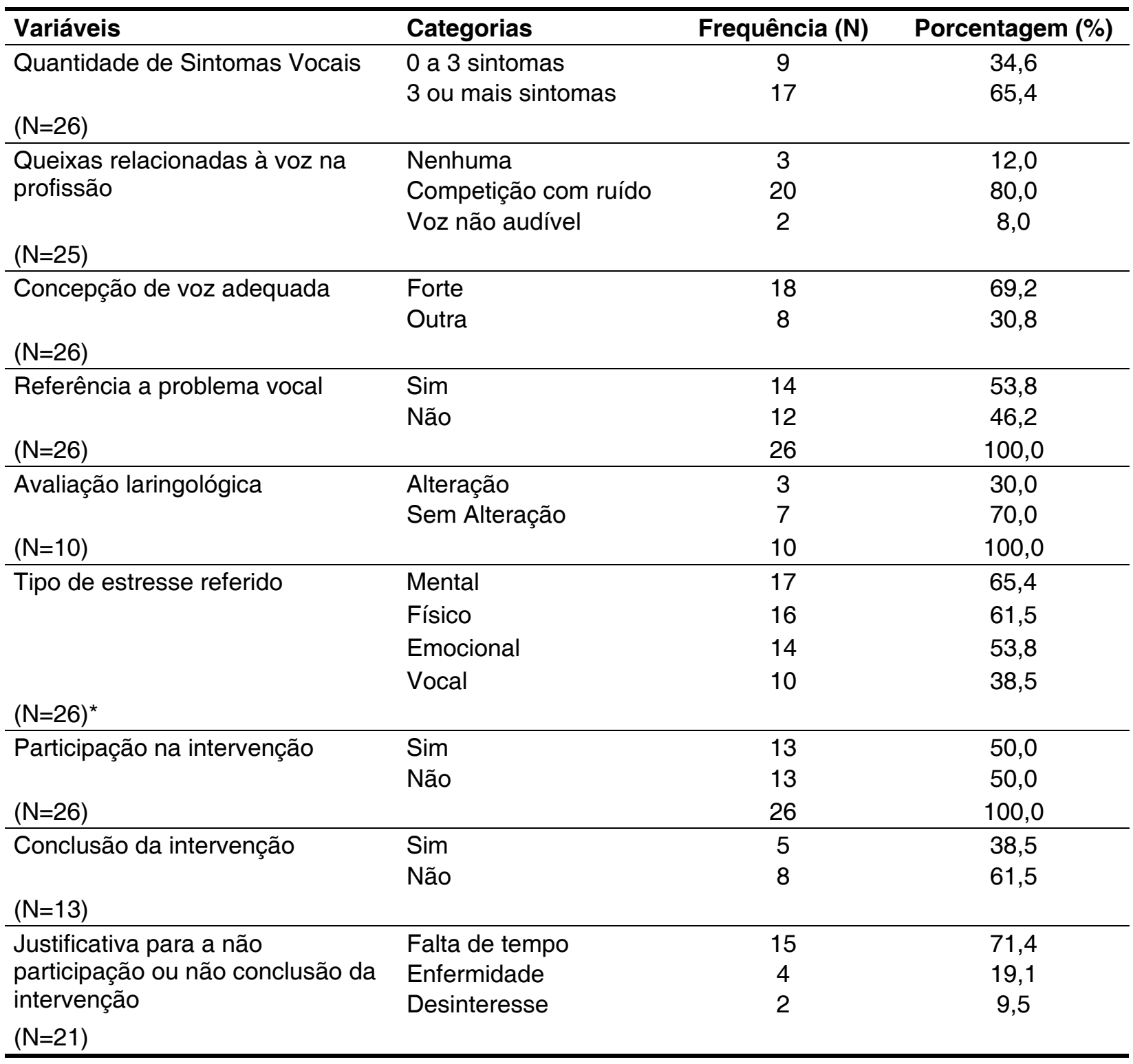

${ }^{*}$ Os sujeitos referiram mais de um tipo de estresse.

a necessidade de intervenções que contribuam para uma maior percepção e reconhecimento da própria voz, suas variações e transformações, e para o reconhecimento da importância da voz no processo ensino/aprendizagem. No entanto, a falta de tempo, principal justificativa dos professores do presente estudo, dificulta a participação em intervenções de médio a longo prazo, se não houver apoio do empregador. O que pôde ser observado na tendência à associação entre a indisponibilidade de tempo e a referencia a estresse vocal encontrada no presente estudo.

Nota-se que $88 \%$ (22) referiram algum tipo de queixa relacionada à voz na profissão. A queixa da maioria estava relacionada à necessidade de elevar a intensidade da voz devido à conversa ou indisciplina dos alunos, e/ou competição com ruídos internos e externos. Pesquisadores acreditam que estudando as práticas pedagógicas utilizadas pelos professores, torna-se possível o desenvolvimento de um trabalho sobre a indisciplina dos alunos $^{18}$. A redução da indisciplina tem como consequência a diminuição de problemas vocais, uma vez que, a indisciplina dos alunos conduz o professor ao aumento da intensidade vocal, e conseqüentemente do esforço fonatório.

Os resultados do presente estudo elencam a importância de ações de promoção à saúde, ou 
Tabela 3 - Grau de associação entre os aspectos vocais, ocupacionais e preventivos investigados

\section{Associações (Exposição x Desfecho)}

Quantidade de sintomas vocais $\mathrm{X}$ referência a problema vocal

Concepção de voz adequada à profissão x quantidade de sintomas vocais

Quantidade de sintomas vocais $x$ participação na intervenção

Referência a estresse vocal $x$ referência a enfermidade

Indisponibilidade de tempo $x$ referência a estresse vocal

Concepção de voz adequada à profissão $\mathrm{x}$ referência a problema vocal

Referência a estresse vocal x participação na intervenção

Concepção de voz adequada à profissão $x$ participação na intervenção

Quantidade de sintomas vocais $x$ conclusão da intervenção

Indisponibilidade de tempo $\mathrm{x}$ quantidade de sintomas vocais

Quantidade de sintomas vocais $x$ enfermidade

Referência a estresse vocal $x$ conclusão da intervenção

Avaliação laringológica $x$ conclusão da intervenção
Grau de Associação

(Teste Qui-Quadrado)

0,133

0,587

$0,048^{*}$

0,167

$0,055^{\text {** }}$

0,246

0,656

0,664

0,094

0,523

0,414

0,274

0,083

*p>0,05; **tendência

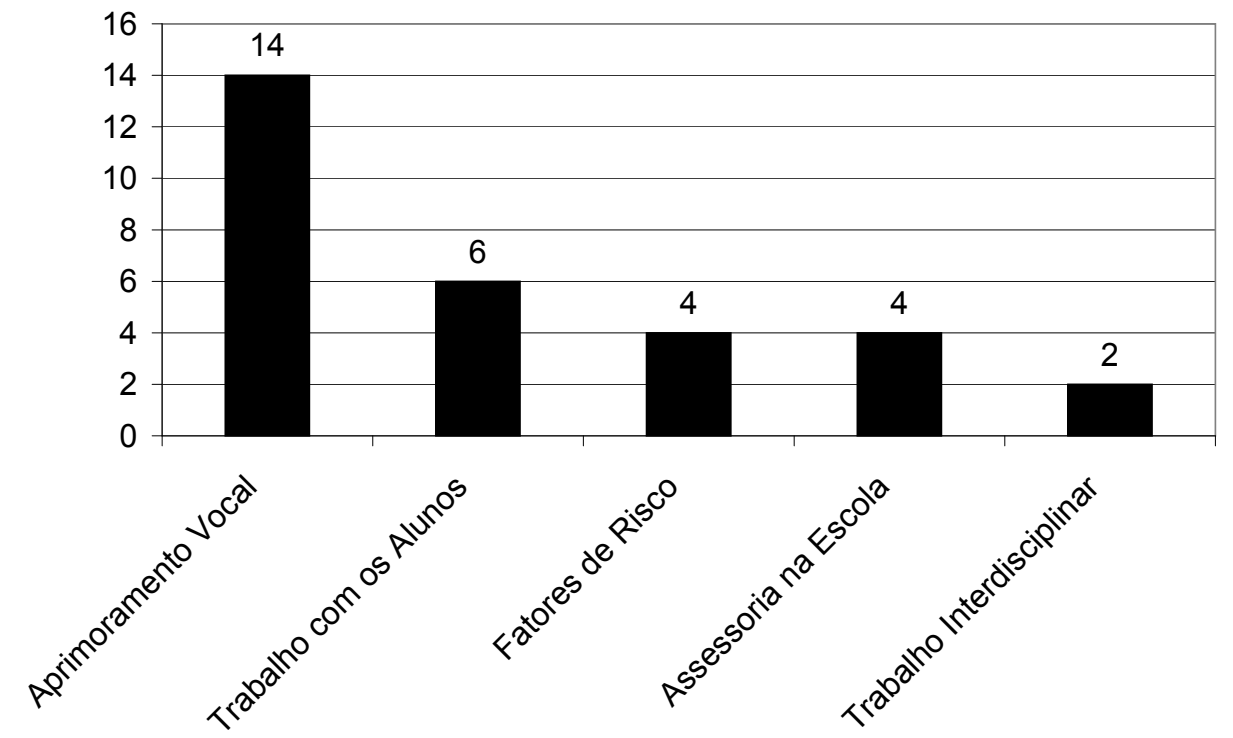

Figura 1 - Frequência de distribuição das sugestões dos professores quanto às ações de prevenção e promoção à saúde vocal

seja, ações que objetivem não apenas a saúde vocal, mas o bem-estar deste profissional como um todo, ações que envolvam todos os atores envolvidos com a escola (alunos, pais, professores e gestores) buscando o bem-estar coletivo. Uma atuação conjunta entre professores, pais e alunos, que faça com que a escola deixe de ser um espaço de culpabilização, do aluno por sua indisciplina ou do professor por suas práticas pedagógicas, para ser um espaço, no qual os atores envolvidos atuem juntos para a construção do saber e da cidadania.
Entretanto, para que estas mudanças sejam possíveis, políticas educacionais, pedagógicas e de promoção da saúde precisam ser implantadas deste o ensino infantil ${ }^{18}$.

O excesso de ruído interno e/ou externo nas escolas também é um fator agravante. Trata-se de um fator de risco para disfonia, pois induz os professores a elevarem a intensidade de suas vozes para tornarem-na audível aos alunos, aumentando o esforço e o cansaço vocal no final do dia. Além disso, o elevado nível de ruído na escola também 
prejudica o aprendizado dos alunos. A Norma Brasileira NBR 10.152 da ABNT ${ }^{19}$, preconiza que o nível de ruído em uma escola seja até $45 \mathrm{dBA}$. Estudo realizado na cidade de Piracicaba, estado de São Paulo, encontrou ruído entre 55dBA e 102dBA nas salas de aula da escola estudada ${ }^{20}$. Faz-se necessária a implantação de medidas que auxiliem na redução do ruído e na melhora das condições acústicas das salas de aula.

Observa-se a importância na construção de um Programa de intervenção com toda a equipe de saúde do trabalhador, na qual cada profissional constitua práticas de sua competência. Para dar início à intervenção, seria interessante um trabalho de sensibilização para a importância da voz no ensino, que proporcionasse ao professor uma reflexão sobre o papel da voz no ensino, o impacto deste instrumento de trabalho sobre a aprendizagem do aluno e o aumento de sua sensibilidade para queixas e sintomas vocais.

Como próximo passo poderia ser identificado os fatores de risco para a saúde dos professores, por meio de visitas as escolas e observação dos professores em atividade letiva. Assim, os efeitos negativos do ambiente de trabalho, da organização do trabalho e de sua execução poderiam ser estudados, atenuados ou eliminados quando necessário.

Como complemento a esta proposta, seria interessante a investigação de ações, como programas de aprimoramento vocal, que visam fornecer subsídios para uma produção vocal mais adequada ao uso vocal intenso, exigido pela profissão.

A menor referência ao estresse vocal, comparada a porcentagem de professores que relataram problemas vocais, pode estar relacionada à falta de sensibilidade na percepção dos sinais de alteração vocal ou ao excesso de tolerância à disfonia. A alta menção a estresse mental, físico e emocional corrobora com a literatura, na qual se observa o aumento da prevalência de transtornos psíquicos nesta população devido às condições objetivas, como falta de material e ambiente físico inadequado, e subjetivas, como relações interpessoais com alunos e/ou gestores ${ }^{22}$.

Diante de todo este contexto, seria interessante a realização de estudos sobre as reais condições de trabalho e levantamento dos principais fatores de risco para a saúde dos professores em cada escola, para que as ações fossem direcionadas efetivamente para a prevenção e a promoção à saúde do professor.

Quando questionados sobre as ações que poderiam auxiliá-los em relação ao uso da voz, os professores sugeriram ações que envolvem questões interdependentes do trabalho, pois têm a visão da prática docente. A maioria das sugestões não é voltada especificamente para os cuidados com voz, no entanto, todas contribuem para a saúde vocal do professor, dado que a voz carrega elementos não apenas orgânicos, mas também psicológicos, ambientais e socioeconômico-culturais ${ }^{21}$. Este olhar multifocal evidencia que, para promover saúde vocal ou prevenir alterações vocais em professores, não bastam atuações voltadas apenas para os cuidados com a voz, são necessárias ações que abordem os aspectos envolvidos na saúde do professor.

Também é importante mencionar a importância de ações educativas em prevenção, estruturadas de modo a mudar hábitos e aprimorar os mecanismos de produção da voz, e estratégias de promoção à saúde que enfatizem mudanças nas condições de vida e trabalho, e apóiem à adoção de políticas públicas que incluam medidas fiscais, taxações e mudanças organizacionais.

O professor tem consciência do importante papel que exerce na sociedade, que ultimamente tem depositado na escola, e não na família, o ato de educar. Faltam ações de saúde coletiva, ou pública, que considerem a coexistência de duas áreas para a prevenção de alterações vocais em professores, a saúde e educação num só contexto de promoção.

É de extrema importância a realização de novos estudos envolvendo outros parâmetros, maior número de sujeitos e outros desenhos metodológicos, visando gerar mais evidências e discussões na temática "voz do professor". Estudos sobre intervenções contextualizadas, que considerem o professor juntamente com as especificidades do contexto de desenvolvimento de seu trabalho, poderão ampliar as chances de prevenir alterações vocais nestes profissionais.

\section{CONCLUSÃO}

Durante muito tempo culpabilizava-se o professor pela alta prevalência de alterações vocais em sua classe profissional. Hoje se sabe que tal alteração não deve ser atribuída apenas à falta de percepção, tolerância à disfonia ou ao uso vocal inadequado. Fatores ambientais e organizacionais são amplamente reconhecidos como contribuintes para o desgaste vocal do professor. Acredita-se que a saúde vocal do professor esteja amplamente relacionada a aspectos ocupacionais, mesmo não tendo sido encontrada significância estatística em todas as associações investigadas neste artigo.

Muitos são os estudos cujo principal objetivo é a voz do professor, no entanto, este problema de saúde coletiva, infelizmente, ainda está longe de ser resolvido. Com base nos resultados do presente 
estudo, elenca-se para a prevenção e promoção da saúde vocal do professor, ações multi ou interdisciplinares que considerem o professor em suas reais condições de trabalho. Trabalhos de sensibilização para a importância da voz no ensino, identificação e atenuação de fatores de risco, programas de aprimoramento vocal e integração entre todos os atores envolvidos com a escola.

\begin{abstract}
Purpose: to examine possible actions for preventing and promoting vocal health of teachers through the understanding of interactions among vocal, occupational and preventive aspects Methods: we developed a qualitative and quantitative study at a public school in the state of Sao Paulo, Brazil, with 26 teachers. This study developed a program to improve voice, which counted on a laryngological examination, a questionnaire application and a preventive intervention-therapy group. To quantify the degree of association between the vocal, occupational and preventive aspects, we used the Chisquare test. Results: it was observed that most teachers (except two) reported some type of complaint related to the use of voice in the profession. Alterations were observed in three of the ten teachers who underwent laryngological examination. Twenty subjects reported some type of stress, and from these, 10 reported vocal stress. Thirteen teachers took part in the intervention group, however, only five completed it. There was a statistically significant association between the quantity of vocal symptoms and participation in the preventive-therapeutic intervention group, showing that teachers with more than three vocal symptoms took part in the group. There was also a trend for association between lack of time and reference to vocal stress. Conclusion: we believe that vocal health of teachers is largely related to occupational aspects, even if we did not find statistically significant associations in all investigated. The preventive actions are to capitalize on the importance of multi-or interdisciplinary performances, on the works to raise awareness of the importance of voice in the education process, on the identification and mitigation of risk factors, on vocal enhancement programs and on voice integration among all actors involved with the school.
\end{abstract}

KEYWORDS: Occupational Health; Disease Prevention; Health Promotion; Working Conditions; Voice; Public Health

\section{REFERÊNCIAS}

1. Instituto Nacional de Estudos e Pesquisas Educacionais (INEP). Disponível em: http://www. inep.gov.br/download/censo/2003/estatisticas_ professores.pdf. Acesso em 13 out 2008.

2. Araújo TM, Reis EJFB, Carvalho FM, Porto LA, Reis IC, Andrade JM. Fatores associados a alterações vocais em professoras. Cad Saúde Pública. 2008; 24(6):1229-38.

3. Jardim R, Barreto SM, Assunção AA. Condições de trabalho, qualidade de vida e disfonia entre docentes. Cad Saúde Pública. 2007;22(10):2439-61.

4. Roy N, Merrill RM, Thibealt S, Parsa RA, Gray SD, Smith EM. Prevalence of voice disorders in teachers and the general population. J Speech Lang Hear Res. 2004; 47:281-93.

5. Smith E, Gray SD, Dove H, Kirchner L, Heras H. Frequency and effects of teachers' voice problems. J Voice. 1997; 11(1):81-7.
6. Simberg S, Sala E, Tuomainen J, Sellman J, Rönnemaa. The effectiveness of group therapy for students with mild voice disorders: a controlled clinical trial. J Voice. 2006; 20(1):97-109.

7. Duffy OM, Hazlett DH. The impact of preventive voice care programs for training teachers: a longitudinal study. J Voice. 2004; 18(1):63-70.

8. Gillivan-Murphy $P$, Drinnan MJ, Dwyer TPO, Rdha $\mathrm{H}$, Carding P. The effectiveness of a voice treatment approach for teachers with self-reported voice problems. J Voice. 2006; 20(3):423-31.

9. Grillo MHMM. The impacto of a vocal improvement course in a speech language and hearing science prevention context. Pro-Fono. 2004; 16(2):159-68.

10. Niebudek-Bogusz E, Sznurowska-Prygocka B, Fiszer M, Kotylo P, Sinkiewicz, Modrzewska M, et al. The effectiveness of voice therapy for teachers with dysphonia. Folia Phoniatr Logop 2008;60:134-41. 
11. Kooijman PGC, Jong FICRS, Thomas G, Huinck W, Donders R, Graamans K, Schutte HK. Risk factors for voice problems in teachers. Folia Phoniatr Logop 2006;58:159-174.

12. Penteado RZ, Pereira IMTB. Qualidade de vida e saúde vocal de professores. Rev Saúde Pública. 2007; 41(2):236-43.

13. Sapir S, Keidar A, Mathers-Schmidt B. Vocal attrition in teachers: survey findings. Eur $\mathrm{J}$ Disord Commun. 1993; 28:177-85.

14. Simões $M$, Latorre MRDO. Prevalência de alteração vocal em educadoras e sua relação com a auto-percepção. Rev Saúde Pública. 2006; 40(6):1013-8.

15. Brasil. Ministério da Saúde. Secretaria de Políticas de Saúde. Projeto Promoção da Saúde. As cartas de promoção da saúde. Brasília: Ministério da Saúde; 2002.

16. Oliveira IB de. Avaliação fonoaudiológica da voz: reflexões sobre condutas, com enfoques à voz profissional. In: Ferreira LP, Befi-Lopes DM, Limongi SCO (orgs). Tratado de Fonoaudiologia. São Paulo: Roca; 2004. p.11-24.
17. Bovo R, Galceran, Petruccelli J, Hatzopoulos S. Vocal problems among teachers: evaluation of a preventive voice program. J Voice 2007; 21(6):705-22.

18. Tuleski SC, Eidt NM, Menechinni AN, Silva EF, Sponchiado D, Colchon PD. Voltando o olhar para o professor: a psicologia e a pedagogia caminhando juntas. Rev Dep Psicol. 2005; 17(1):129-37.

19. Brasil. Associação Brasileira de Normas Técnicas. NBR 10.152: Níveis de ruído para conforto acústico. Rio de Janeiro; 1987.

20. Libardi A, Gonçalves CGO, Vieira TPG, Silverio KCA, Rossi D, Penteado RZ. O ruído em sala de aula e a percepção dos professores de uma escola de ensino fundamental de Piracicaba. Rev Dist Comun. 2006; 18(2):167-78.

21. Behlau M, Madazio G, Pontes P. Disfonias organofuncionais. In: Behlau M, organizador. Voz: o livro do especialista. Vol.1. Rio de Janeiro: Revinter; 2004. p. 295-329.

22. Gasparini SM, Barreto SM, Assunção AA. O professor, as condições de trabalho e os efeitos sobre sua saúde. Educ Pesq. 2005; 31(2):189-99.

DOI: 10.1590/S1516-18462010005000112

RECEBIDO EM: 11/12/2009

ACEITO EM: 19/03/2010

Endereço para correspondência:

Lucia Figueiredo Mourão

Centro de Estudos e Pesquisas em Reabilitação

(CEPRE/FCM/UNICAMP)

Av. Tessália Vieira de Camargo, 126

Campinas - SP

CEP: 13083-887

E-mail: lumourao@fcm.unicamp.br 